\title{
Mechanisms by which the N-terminal 24 amino acids of the p55 regulatory subunit of phosphatidylinositol 3-kinase affect endotoxin-induced cytokine release in human keratinocytes
}

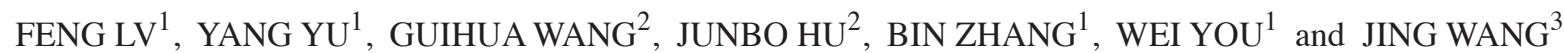 \\ ${ }^{1}$ Department of Breast Surgery, Henan Provincial People's Hospital, The People's Hospital of Zhengzhou University, \\ Zhengzhou, Henan 450003; Departments of ${ }^{2}$ Molecular Medical Center and ${ }^{3}$ Immunology, Tongji Hospital, \\ Huazhong University of Science and Technology, Wuhan, Hebei 430030, P.R. China
}

Received April 18, 2014; Accepted December 12, 2014

DOI: $10.3892 / \mathrm{mmr} .2015 .3184$

\begin{abstract}
To understand the association between the cytokine network and psoriasis, the present study cultured human keratinocytes (HaCaT cells) and investigated the effects of the phosphatidylinositol-3-kinase (PI3K) p55 regulatory subunit (p55PIK), and its N-terminal 24 amino acids (N24) on the regulation of endotoxin (LPS)-induced cytokine secretion. The results of the enzyme-linked immunosorbent assay and reverse transcription quantitative polymerase chain reaction revealed an increased release of the inflammatory cytokines, tumor necrosis factor (TNF)- $\alpha$, interleukin (IL) -6 and IL-8 in the HaCaT cells following LPS stimulation. Transfection with the adenovirus (AD)-N24-green fluorescent protein (GFP) suppressed the release of these cytokines, whereas AD-p55PIK-GFP increased their release. Immunocytochemistry detected a low level of nuclear factor (NF)- $\kappa$ B p65 staining in quiescent HaCaT cells, which was localized primarily in the cytoplasm. LPS stimulation induced the translocation of $\mathrm{NF}-\kappa \mathrm{B}$ p 65 protein into the nucleus and intense staining suggested increased expression. Transfection with AD-N24-GFP reduced the expression of $\mathrm{NF}-\kappa \mathrm{B}$ p65 in the nucleus. Western blot analysis demonstrated that AD-N24-GFP downregulated the expression levels of the Toll-like receptor (TLR)2/TLR4/myeloid differentiation factor 88 (MyD88) pathway components in the HaCaT cells, without affecting the PI3K/Akt signaling pathway.
\end{abstract}

Correspondence to: Dr Jing Wang, Department of Immunology, Tongji Hospital, Huazhong University of Science and Technology, 1095 Jiefang Road, Wuhan, Hebei 430030, P.R. China

E-mail:wangjhxh@163.com

Dr Bin Zhang, Department of Breast Surgery, Henan Provincial People's Hospital, The People's Hospital of Zhengzhou University, 7 Weiwu Road, Zhengzhou, Henan 450003, P.R. China

E-mail: zhangbin841919@gmail.com

Key words: human keratinocytes, phosphatidylinositol-3-kinase, interleukin, endotoxin, Toll-like receptors
Transfection with AD-p55PIK-GFP resulted in an increased expression level of MyD88 protein and phosphorylated Akt. Co-transfection with AD-N24-GFP and AD-p55PIK-GFP did not significantly alter the levels of phosphorylated extracellular-signal-regulated kinases $1 / 2$, c-Jun N-terminal kinases or p38, indicating that AD-N24-GFP and AD-p55PIK-GFP did not affect the mitogen-activated protein kinase signaling pathway. In conclusion, AD-N24-GFP effectively inhibited the LPS-induced expression levels of TNF- $\alpha$, IL- 6 and IL-8. The elevated expression of p55PIK synergized with LPS and promoted the release of inflammatory cytokines. AD-N24-GFP and AD-p55PIK-GFP affected LPS-induced inflammatory cytokine release in the $\mathrm{HaCaT}$ cells through the TLRs/MyD88 signaling pathways.

\section{Introduction}

Autoimmunity is the failure of an organism to recognize its own constituents as self, which causes an immune response against its own cells and tissues. Any disease resulting from this aberrant immune response is termed autoimmune disease (AID) (1). AID is frequently observed clinically and therapy for AID often includes treatment with hormones and immunosuppressants (1). These agents are effective in the short-term, however, long-term use increases the incidence of tumors and uncontrolled infectious diseases due to various side effects $(1,2)$. Therefore, AID is recognized worldwide as a '5D' disease, which comprises discomfort, disability, drug toxicity, dollar loss and death (3).

Psoriasis is a common AID and the primary pathological features include the abnormal proliferation of epidermal cells and infiltration of inflammatory cells. Although the exact pathogenesis of psoriasis has not been fully elucidated, the importance of $\mathrm{T}$ cells has been demonstrated in previous studies (4,5). Cytokines within psoriatic lesions, including tumor necrosis factor (TNF)- $\alpha$, interleukin (IL)-1, IL-6 and IL-8 (6-8), are subject to complex interactions and regulation, suggesting their importance in the development of this inflammatory disease. Therefore, understanding the interactions involved in the cytokine network has been a major area of focus in the investigation of the pathogenesis of psoriasis (9). In the present 
study, to further understand the association between the cytokine network and psoriasis, human keratinocytes (HaCaT cells) were cultured in vitro $(10,11)$ to investigate the effects of the $\mathrm{N}$-terminal 24 amino acids (N24) and the expression of the p55 regulatory subunit of phosphatidylinositol 3-kinase (p55PIK) on the regulation of endotoxin (LPS)-induced cytokine secretion.

\section{Materials and methods}

Cells and plasmids. The HaCaT cell line was purchased from the American Type Culture Collection (Manassas, VA, USA) and was subcultured in the laboratory and stored at $-80^{\circ} \mathrm{C}$. The cells were cultured in Dulbecco's modified Eagle's medium (DMEM; Gibco Life Technologies, Carlsbad, CA, USA) containing 15\% newborn calf serum (Evergreen, Hangzhou, China) and $100 \mu \mathrm{g} / \mathrm{ml}$ penicillin and streptomycin (Evergreen) and incubated at $37^{\circ} \mathrm{C}$ with $5 \% \mathrm{CO}_{2}$. The $\mathrm{N} 24$ and p55PIK adenoviruses (Evergreen) were purified and stored in the laboratory as previously described (12).

Reagents. Trypsin was purchased from Gibco Life Technologies, LPS (<0.008\% protein from E. coli K235) was purchased from Sigma-Aldrich (St. Louis, MO, USA) and the TNF- $\alpha$ and IL enzyme-linked immunosorbent assay (ELISA) kits were purchased from Westang Biotechnology (Shanghai, China).

Cryopreservation of the HaCaT cells and in vitro culture. The HaCaT cells stored in cryovials (Biyuntian Health Care Products Co., Hangzhou, China) were removed from the $-80^{\circ} \mathrm{C}$ freezer and rapidly thawed by submersing the cryovial in a $37^{\circ} \mathrm{C}$ water bath. The cryovial tubes were opened in a laminar flow hood and the cell suspension was transferred into centrifuge tubes and centrifuged (Beckman Coulter, Brea, CA, USA) at a low speed (100 x g for $5 \mathrm{~min}$ ). The supernatant was discarded and the cell pellet was gently resuspended in DMEM containing $15 \%$ newborn calf serum and $100 \mu \mathrm{g} / \mathrm{ml}$ penicillin and streptomycin and transferred into a culture flask (Biyutian Health Care Products Co.). The cells were cultured in a $37^{\circ} \mathrm{C}$ thermostatic incubator with $5 \% \mathrm{CO}_{2}$ and the medium was replaced every 2-3 days until a confluent monolayer had formed. The cells were washed three times with D-Hanks solution (Biyutian Health Care Products Co.) prior to the addition of $1 \mathrm{ml}$ of $0.25 \%$ trypsin and incubated for $10 \mathrm{~min}$ at $37^{\circ} \mathrm{C}$ in a $5 \% \mathrm{CO}_{2}$ atmosphere. DMEM $(10 \mathrm{ml})$ was added to terminate the digestion upon detachment of the adherent cells from the bottom of the flask. The cells were gently dissociated by pipetting up and down into a single cell suspension and $1 \times 10^{4}$ cells were transferred into a new flask. The medium was replaced every 2-3 days and the cells were passaged every $\sim 6$ days. Viable cells with a healthy morphology were used for experiments.

ELISA detection of inflammatory cytokine secretion by HaCaT cells. The following groups were evaluated: Blank control, LPS-stimulation, adenovirus (AD) expressing-green fluorescent protein (GFP), AD-N24-GFP, AD-p55PIK-GFP, AD-GFP+LPS, AD-N24-GFP+LPS and AD-p55PIK-GFP+LPS. Viable cells were seeded into six-well plates, incubated at $37^{\circ} \mathrm{C}$ and transduced with AD-GFP, AD-N24-GFP or AD-p55PIK-GFP viruses until the cells reached $70 \%$ confluence. The transduction efficiency multiplicity of infection value was set to 100 and the cells were further cultured for $24 \mathrm{~h}$ at $37^{\circ} \mathrm{C}$. The cells were stimulated using LPS $(10 \mu \mathrm{l})$ at a final concentration of $100 \mathrm{ng} / \mathrm{ml}$ for $4 \mathrm{~h}$, and the culture supernatants were collected and stored at $-80^{\circ} \mathrm{C}$ until use. The assays were performed within two weeks.

Reverse transcription quantitative polymerase chain reaction (RT-qPCR) to assess the effects of AD-GFP, AD-N24-GFP and AD-p55PIK-GFP on the mRNA expression levels of TNF- $\alpha$, IL-6 and IL-8 mRNA in LPS-stimulated HaCaT cells. The primers for RT-qPCR were synthesized by Invitrogen Biotechnology Co., Ltd. (Shanghai, China), based on the receptor mRNA template from the GenBank nucleotide sequence database (TNF- $\alpha$, NM000594.2, 8 July 2012; IL-6, NM000600.3, 11 May 2014 and IL-8 NM000584.2, 6 February 2011). The primers were purified using a PAGE method (Invitrogen Biotechnology Co., Ltd) and the following primer sequences were used: TNF- $\alpha$, forward 5'-CGAGTGACAAGCCTGTAGCC-3' and reverse 5'-TGAAGAGGACCTGGGAGTAGAT-3'; IL-6, forward 5'-AGCCACTCACCTCTTCAGAACG-3' and reverse 5'-TGCCTCTTTGCTGCTTTCACA-3'; IL-8, forward 5'-CTCCAAACCTTTCCACCCC-3' and reverse 5'-TCCACAACCCTCTGCACCC-3' and GAPDH, forward 5'-GATTTGGTCGTATTGGGCG-3' and reverse 5'-CCTGGAAGATGGTGATGGG-3'. To ensure the consistency of the RNA samples, GAPDH was used as an internal control. The total RNA from the HaCaT cells was extracted using TRIzol Total RNA Isolation reagent (Tiangen Biotech Co., Beijing, China) according to the manufacturer's instructions. RT-qPCR was performed based on the features of the primers and the conditions for the PCR reactions were determined from pilot experiments using a SYBR Green mix (Tiangen Biotech Co., Beijing, China), according to the manufacturer's instructions. The PCR results were analyzed for the target gene expression by the $2^{-\Delta \Delta \mathrm{CT}}$ method. In this formula, the cycle threshold (CT) is defined as the number of cycles required for the fluorescent signal to cross the threshold and exceed the background level. $\Delta \Delta \mathrm{CT}=$ (experimental group $\mathrm{CT}$ target gene - CT housekeeping gene) - (control group $\mathrm{Ct}$ target gene - $\mathrm{Ct}$ housekeeping gene). Therefore, $2^{-\Delta \Delta C T}$ represents the fold-change of the target gene in the experimental group relative to the control group. This method directly calculates the levels of the target gene expression relative to those of the housekeeping gene.

Immunohistochemistry assay for nuclear factor $(N F)-\kappa B$ activity. Viable cells were cultured on cover glasses and subjected to immunohistochemical staining with a Diaminobenzidine Histochemistry kit (Biyuntian Health Care Products Co.) to detect NF- $\kappa \mathrm{B}$ p65-positive cells. NF- $\kappa \mathrm{B}$ p65-positive cells were detected based on brown staining in the cell nuclei. To calculate the numbers of positive cells, five regions from each cover glass (Tiangen Biotech Co., Beijing, China) were randomly selected under a magnification of x200 (Leica DMIL 090-135.002; Leica Microsystems GmbH, Wetzlar, Germany). The positive cell ratio was calculated by comparing the number of positively stained cells with the total number of cells. Positive cell ratio $(\%)=$ number of positive cells / number of total cells x $100 \%$. 
Western blot analysis to detect the protein expression levels of the Toll-like receptor (TLR)2/TLR4/myeloid differentiation factor 88 (MyD88)/PI3K/Akt and mitogen-activated protein kinase (MAPK) signaling pathways and of the I $\kappa B-\alpha N F-\kappa B$ inhibitor. The expression levels of these proteins were monitored in the HaCaT cells expressing either AD-N24-GFP or AD-p55PIK-GFP at different time-points following LPS stimulation to confirm the timing of the peak expression. The proteins were extracted, according to the instructions provided by Santa Cruz Biotechnology, Inc. (Dallas, TX, USA) and bovine serum albumin Bio-Rad Laboratories, Inc., Hercules, CA, USA) was used as the standard. The cells from all the groups were lysed in cold NP-40 protein lysis buffer [50 mM Tris (pH 7.4), $150 \mathrm{mM} \mathrm{NaCI}, 0.5 \%$ NP-40, $20 \%$ glycerol with $1 \mathrm{mM}$ dithiothreitol, $1 \mathrm{mM}$ phenylmethylsulfonyl fluoride, leupeptin and pepstatin (10 g/mI each) and $100 \mathrm{pg} / \mathrm{mI}$ aprotinin] for $20 \mathrm{~min}$. Lysates were clarified by centrifugation at $10,000 \mathrm{x} \mathrm{g}$ and $4^{\circ} \mathrm{C}$ for $20 \mathrm{~min}$. The standard curve for protein concentration was calculated using a Bio-Rad Protein Assay kit (Bio-Rad Laboratories, Inc.) according to the manufacturer's instructions. The absorbance values were detected at $595 \mathrm{~nm}$ on a spectrophotometer (NanoDrop1000; Thermo Fisher Scientific, Wilmington, DE, USA) and the target protein concentration was calculated based on the standard curve. The total protein $(20 \mu \mathrm{g})$ was loaded onto a $12 \%$ polyacrylamide gel for electrophoresis and then transferred onto a polyvinylidene difluoride membrane at $100 \mathrm{~V}$ for $1 \mathrm{~h}$. Primary antibodies (Santa Cruz Biotechnology, Inc.) were diluted 1:1,000 in PBS and added to the membrane following blocking with 5\% skim milk. GAPDH was used as a loading control. The antibodies used were as follows: rabbit polyclonal immunoglobulin $\mathrm{G}(\mathrm{IgG})$ anti-NFкB p65 (C-20; sc-372); rabbit polyclonal IgG anti-TLR2 (H-175; sc-10739); rabbit polyclonal IgG anti-TLR4 (H-80; sc-10741); rabbit polyclonal IgG anti-MyD88 (HFL-296; sc-11356); rabbit polyclonal IgG anti-Akt1/2/3 (H-136; sc-8312); rabbit polyclonal IgG anti-p-Akt1/2/3 (Ser473; sc-7985)-R; rabbit polyclonal IgG anti-ERK 1/2 (H-72; sc-292838); goat polyclonal IgG p-ERK 1/2 (Thr202/Tyr204; sc-16982); rabbit IgG JNK (FL; sc-571); rabbit polyclonal IgG p-JNK (Thr183; sc-135642); rabbit polyclonal IgG GAPDH (FL-335; sc-25778); rabbit polyclonal IgG p38 (H-147; sc-7149) and rabbit polyclonal IgG p-p38 (Tyr182; sc-101759). Horseradish peroxidase-conjugated secondary antibodies (diluted 1:5,000; donkey anti-goat IgG-HRP, sc-2020, and goat anti-rabbit IgG-HRP, sc-2004; Santa Cruz Biotechnology, Inc.) and an enhanced chemiluminescence detection kit (SuperSignal West Femto Maximum Sensitivity Substrate Trial kit; Pierce Biotechnology, Inc., Thermo Fisher Scientific, Rockford, IL, USA) were used to detect the hybridization signal.

Statistical analysis. The statistical significance was assessed using SPSS 11.5 software (SPSS, Inc., Chicago, IL, USA) and the date represent the mean \pm standard deviation. Differences between the groups were analyzed using unpaired two-tailed Student's t-tests. $\mathrm{P}<0.05$ was considered to indicate a statistically significant difference.

\section{Results}

Effects of AD-N24-GFP and AD-p55PIK-GFP on LPS-induced inflammatory cytokine release by $\mathrm{HaCaT}$ cells. ELISA was
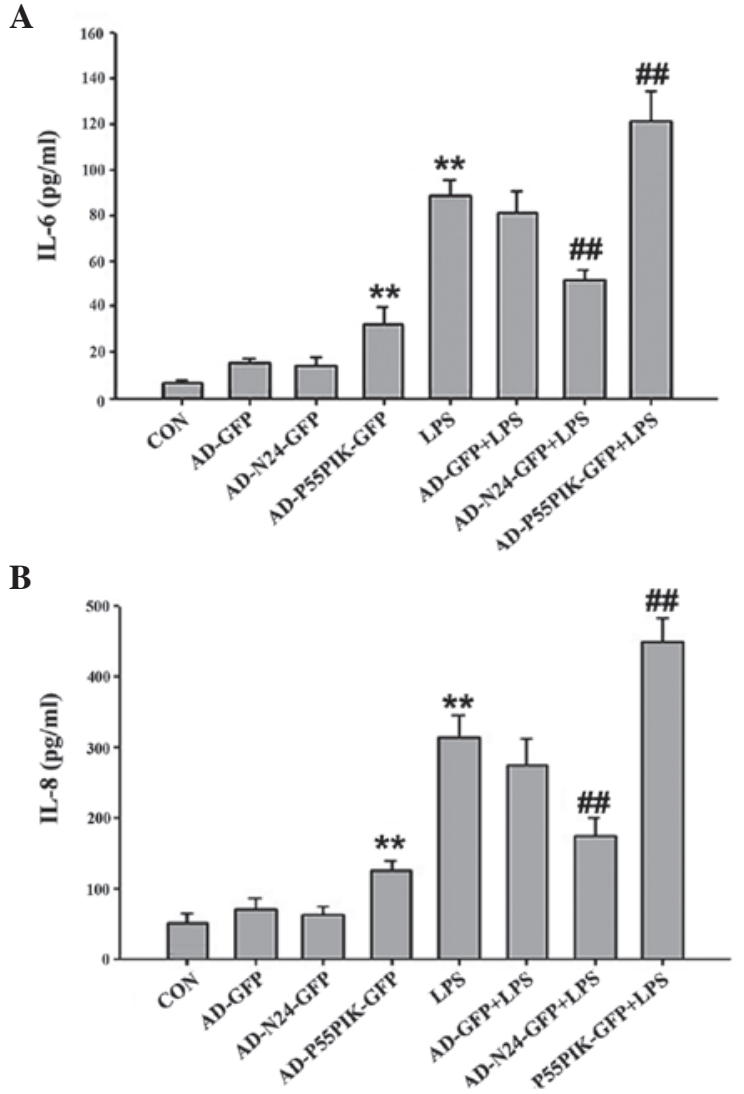

Figure 1. Enzyme-linked immunosorbent assay results indicating the effect of AD-N24-GFP and AD-p55PIK-GFP on the LPS-stimulated release of the (A) IL-6 and (B) IL-8 inflammatory factors $\left({ }^{* *} \mathrm{P}<0.01\right.$, compared with the control group; ${ }^{\# \#} \mathrm{P}<0.01$, compared with the LPS group). LPS, endotoxin; IL, interleukin, GFP, green fluorescent protein, CON, control, N24, N-terminal 24 amino acids; AD, adenovirus. Values are expressed as the mean \pm standard error of the mean of three independent experiments.

used to measure changes in the release of the inflammatory cytokines, IL-6 and IL-8, upon LPS-stimulation following AD-N24-GFP or AD-p55PIK-GFP transfection in the HaCaT cells. IL-6 and IL- 8 were detected in quiescent cells $(6.44 \pm 0.95$ and $50.97 \pm 13.14 \mathrm{pg} / \mathrm{ml}$, respectively), however, the levels of IL-6 and IL-8 in the culture supernatant increased to different extents following LPS-stimulation for $4 \mathrm{~h}(88.32 \pm 7.43$ and $314.57 \pm 30.82 \mathrm{pg} / \mathrm{ml}$, respectively). A more marked increase was observed in the expression of IL-8 compared with IL-6, although statistically significant differences were observed for each protein $(\mathrm{P}<0.05)$. Upon transfection with AD-N24-GFP, the LPS-induced release of IL- 6 and IL- 8 by the HaCaT cells decreased to $51.25 \pm 4.79$ and $174.61 \pm 26.02 \mathrm{pg} / \mathrm{ml}$, respectively. These differences were statistically significant $(\mathrm{P}<0.05)$. Upon transfection with AD-p55PIK-GFP, the expression levels of IL- 6 and IL- 8 increased to $120.99 \pm 13.74$ and $450.28 \pm 32.2 \mathrm{pg} / \mathrm{ml}$, respectively. These differences were also statistically significant $(\mathrm{P}<0.05)$. The data are shown in Fig. 1.

RT-qPCR to determine the LPS-induced mRNA expression of $T N F-\alpha, I L-6$ and IL- 8 by HaCaT cells prior to and following AD-N24-GFP or AD-p55PIK-GFP transfection. Compared with the unstimulated group, LPS-stimulation led to an almost five-fold increase in the mRNA expression levels of TNF- $\alpha$, IL-6 and IL-8 following transfection with AD-N24-GFP. This 


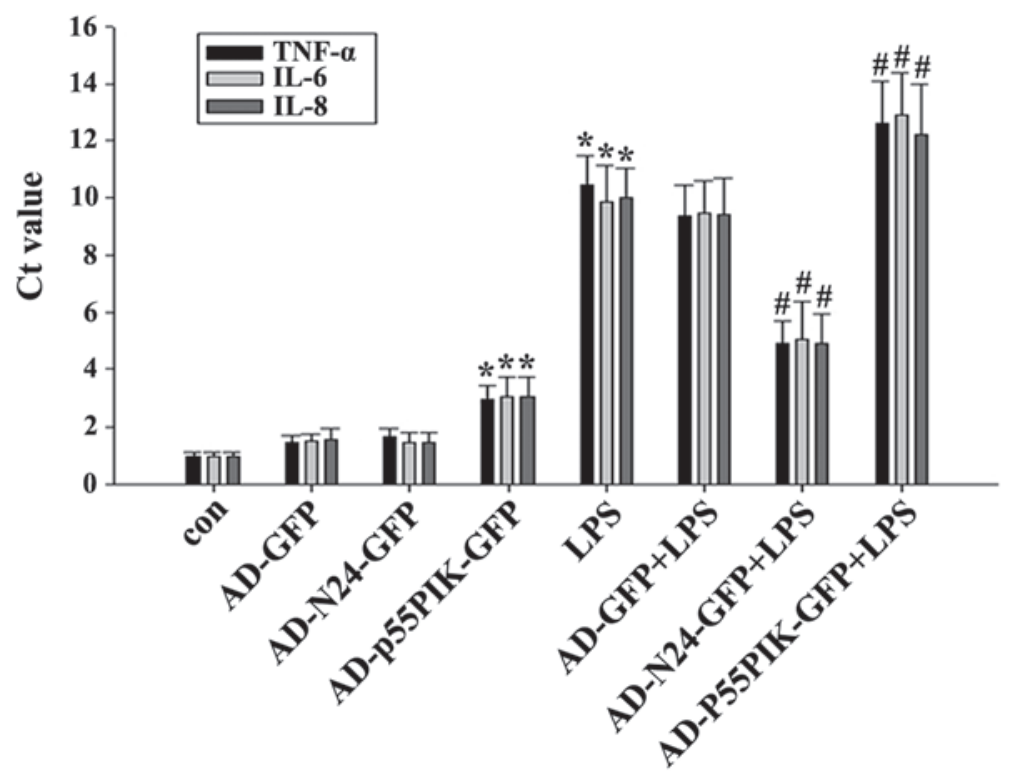

Figure 2. Reverse transcription quantitative polymerase chain reaction detection of the mRNA expression levels of TNF- $\alpha$, IL-6 and IL- 8 in the HaCaT cells $\left(\mathrm{P}<0.05\right.$, compared with the control group; ${ }^{~} \mathrm{P}<0.05$, compared with the LPS group). Ct, cycle threshold; con, control; GFP, green fluorescent protein; LPS, endotoxin; IL, interleukin, N24, N-terminal 24 amino acids; P55PIK, p55 regulatory subunit of phosphatidylinositol 3-kinase; TNF, tumor necrosis factor; $\mathrm{HaCaT}$, human keratinocytes; $\mathrm{AD}$, adenovirus. Values are expressed as the mean \pm standard error of the mean of three independent experiments.

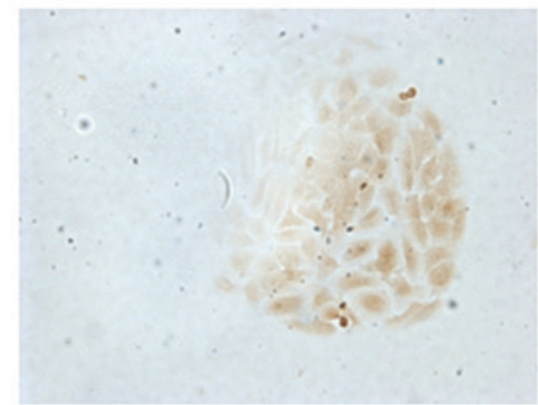

CON

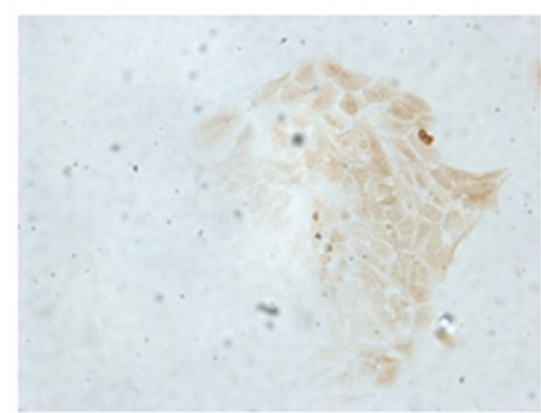

AD-N24-GFP

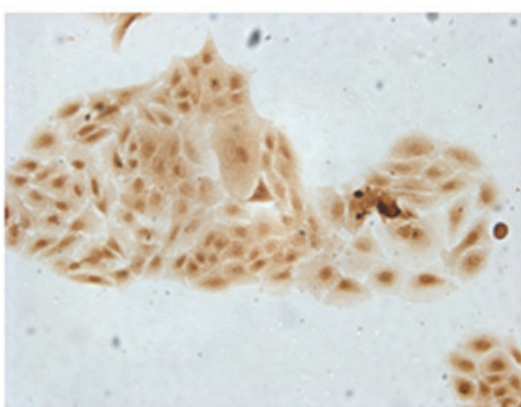

LPS

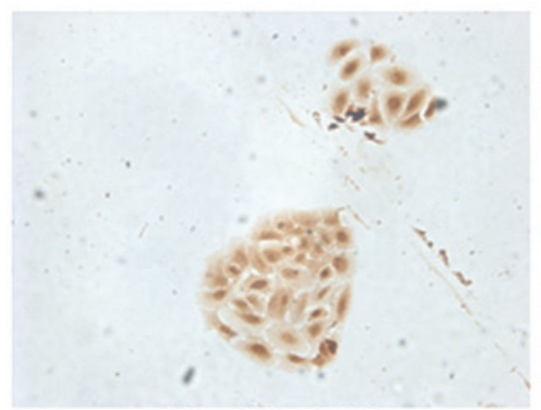

AD-GFP+LPS

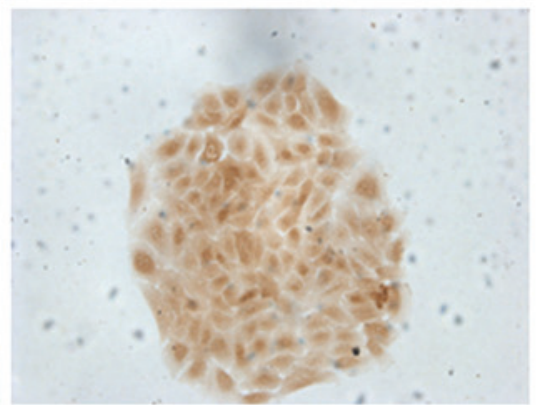

AD-N24-GFP+LPS

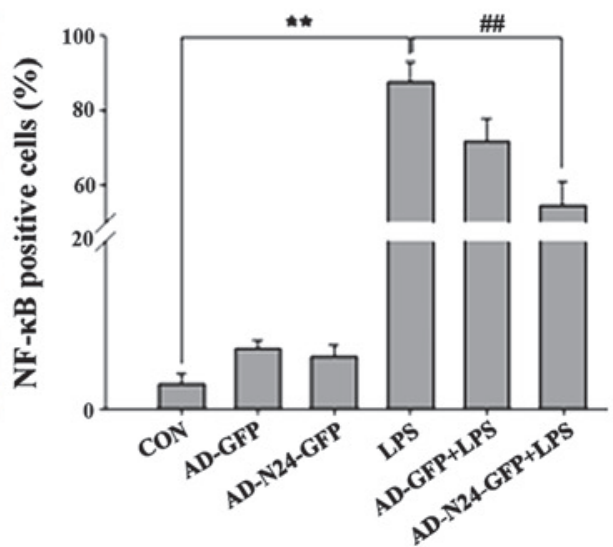

Figure 3. Immunocytochemical analysis of the protein expression of nuclear factor- $\kappa \mathrm{B}$ p65 in the HaCaT cells treated with LPS and AD-N24. The cells were stained with diaminobenzadine (magnification, $\mathrm{x} 200)$. $\left({ }^{* *} \mathrm{P}<0.01\right.$, compared with the control group; ${ }^{\# \#} \mathrm{P}<0.01$, compared with the LPS group). CON, control; GFP, green fluorescent protein; LPS, endotoxin; N24, N-terminal 24 amino acids; HaCaT, human keratinocytes; AD, adenovirus.

was significantly lower compared with that of the untransfected group in response to LPS-stimulation. The mRNA expression levels of the inflammatory cytokines TNF- $\alpha$, IL-6 and IL-8 induced by LPS-stimulation were significantly increased upon transfection with AD-p55PIK-GFP, indicating that an increase in the intracellular expression of p55PIK promoted inflammatory cytokine release. The results are shown in Fig. 2.

Immunocytochemistry to detect the expression of $N F-\kappa B$. $\mathrm{HaCaT}$ cells were cultured on cover glass, fixed and 


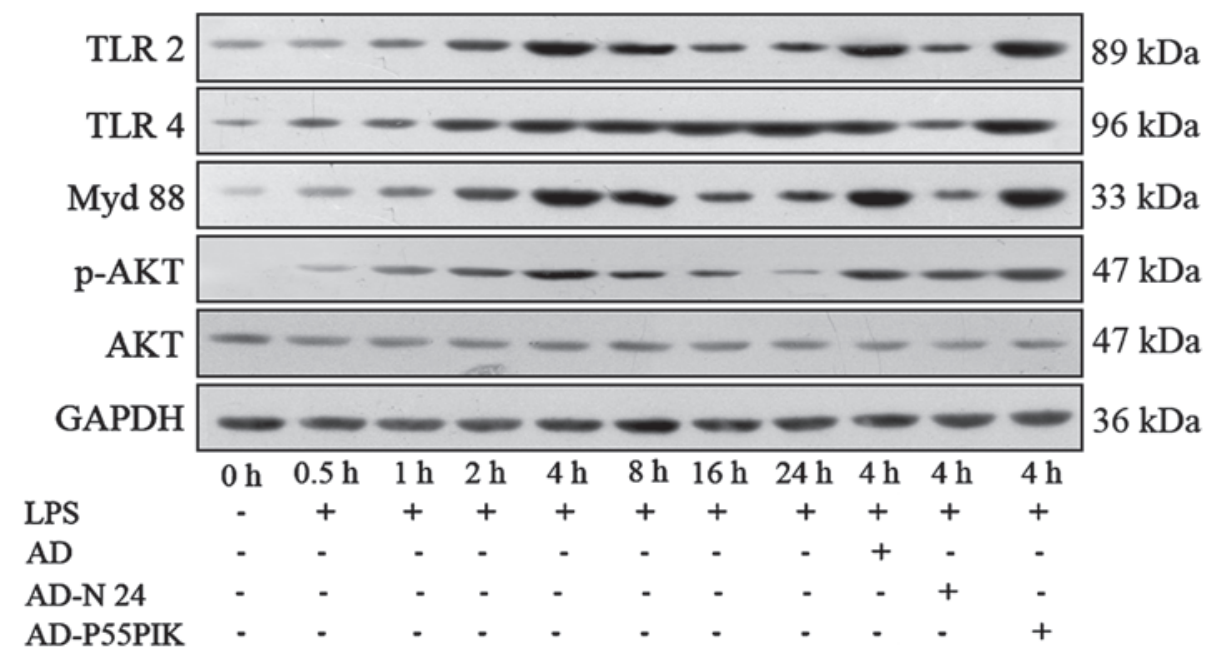

Figure 4. Effect of N24 and p55PIK on the expression levels of the TLRS/MyD88/Akt protein signaling pathway. LPS, endotoxin; TLR, Toll-like receptor; MyD88, myeloid differentiation factor 88; N24, N-terminal 24 amino acids; P55PIK, p55 regulatory subunit of phosphatidylinositol 3-kinase; AD, adenovirus.

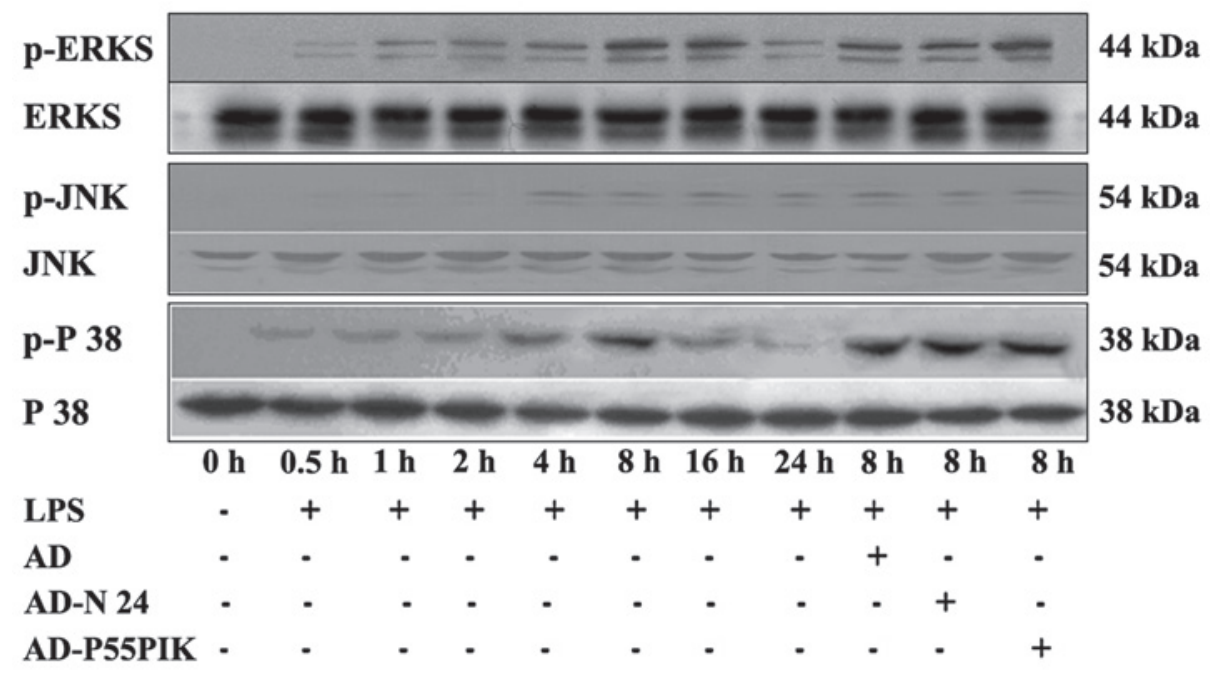

Figure 5. Effect of N24 and p55PIK on the expression of the mitogen-activated protein kinase signaling pathway. ERK, extracellular-signal-regulated kinases; JNK, c-Jun N-terminal kinases; p-, phosphorylated; LPS, endotoxin; N24, N-terminal 24 amino acids; P55PIK, p55 regulatory subunit of phosphatidylinositol 3-kinase; AD, adenovirus.

immunocytochemistry was performed. The numbers of LPS-induced NF- $\mathrm{NB}$ p65-positive $\mathrm{HaCaT}$ cells prior to and following transfection with AD-N24-GFP were counted under a microscope. The quiescent $\mathrm{HaCaT}$ cells contained few $\mathrm{NF}-\kappa \mathrm{B}$ p65-positive cells $(3.04 \pm 1.13 \%)$ compared with the LPS stimulated group, in which LPS almost fully activated NF- $\kappa$ B p65 $(87.57 \pm 5.38 \%)$. Following transfection with AD-N24-GFP, the number of NF- $\kappa \mathrm{B}$ p65-positive HaCaT cells decreased to $54.43 \pm 6.54 \%$, which was statistically significant compared with the LPS group $(\mathrm{P}<0.01)$. The results are shown in Fig. 3.

Protein expression analyses of the TLR signaling pathways in the LPS-simulated HaCaT cells expressing AD-N24-GFP or AD-p55PIK-GFP. The expression levels of the TLR2/TLR4/MyD88/PI3K/Akt signaling pathway proteins were investigated. As shown in Fig. 5, TLR2 and TLR4 were expressed in the HaCaT cells. LPS-stimulation increased the expression levels of TLR2 and TLR4 in the HaCaT cells, with the peak increase observed $4 \mathrm{~h}$ and $8 \mathrm{~h}$ post-stimulation. These levels remained higher than normal for at least $24 \mathrm{~h}$. The expression of MyD88 gradually increased following LPS-stimulation, peaked at $4 \mathrm{~h}$ and then gradually decreased, however remained higher compared with the normal level at $24 \mathrm{~h}$. The phosphorylation of Akt gradually increased $(\mathrm{P}<0.05) 30 \mathrm{~min}$ after LPS-stimulation, peaked at $4 \mathrm{~h}(\mathrm{P}<0.05)$ and then decreased rapidly and returned to normal levels by $24 \mathrm{~h}$. Transfection with AD-GFP had no affect on the expression of the proteins, whereas transfection with AD-N24-GFP significantly downregulated the protein expression levels of TLR2 and TLR4 following LPS stimulation compared with the LPS-stimulated group. The protein expression of MyD88 also decreased and no change in the expression of phosphorylated Akt was observed, suggesting that the AD-N24-GFP protein downregulated the expression levels of TLR2/TLR4/MyD88 in the HaCaT cells with no significant effect on the PI3K/Akt signaling pathway. Transfection with 
AD-p55PIK-GFP increased the protein expression levels of TLR2, TLR4 (to an extent), MyD88 and phosphorylated Akt following LPS stimulation (Fig. 4). These results suggested that the AD-p55PIK-GFP affected the protein expression levels of TLR2/TLR4/MyD88 by upregulating phosphorylated Akt in the $\mathrm{HaCaT}$ cells.

Expression of MAPK signaling pathway proteins. The MAPK signaling pathway is important in the LPS-mediated cellular response. In mammalian cells, at least four MAPK subfamilies have been identified, namely the extracellular-signal-regulated kinases (ERK)1/2, c-Jun N-terminal kinases (JNK), p38 and NIMA-associated protein kinase 5. As shown in Fig. 5, the present study demonstrated that the phosphorylation of ERK increased $30 \mathrm{~min}$ post-LPS stimulation $(\mathrm{P}<0.05)$, peaked at $8 \mathrm{~h}$ $(\mathrm{P}<0.05)$ and then gradually declined, however, it remained significantly higher compared with the baseline until $24 \mathrm{~h}$ $(\mathrm{P}<0.05)$. The phosphorylation patterns of JNK and p38 were similar, however, the phosphorylation of p38 decreased rapidly subsequent to peaking at $8 \mathrm{~h}(\mathrm{P}<0.05)$ and almost returned to basal levels at $24 \mathrm{~h}$. The expression of phosphorylated JNK decreased subsequent to peaking at $4 \mathrm{~h}(\mathrm{P}<0.05)$ and remained marginally higher compared with basal levels at $24 \mathrm{~h}$ (Fig. 5). Transfection with either AD-GFP, AD-N24-GFP or AD-p55PIK-GFP caused no affect on the expression levels of phosphorylated ERK1/2, JNK or p38, suggesting that AD-N24-GFP and AD-p55PIK-GFP had no significant affect on the MAPK signaling pathway.

\section{Discussion}

The skin is the body's first line of defense against outside pathogens and is important in innate immunity. The differentiation features of $\mathrm{HaCaT}$ cells and normal human keratinocytes are similar (13) and have been widely used to investigate the activity and functionality of anti-inflammatory drugs (14). TLRs are primarily expressed in immune cells, including dendritic cells, lymphocytes and monocytes/macrophages. In addition, TLRs are expressed in epithelial cells and other non-immune cells (15). TLR signaling consists of at least two distinct pathways: The MyD88-dependent and the MyD88-independent pathways. MyD88 is essential in intracellular signal transduction through TLRs. The C-terminus of MyD88 contains a Toll/interleukin-1 receptor (TIR) domains, which binds to the TIR domain of TLR, IL-1R and IL-18R. The N-terminus contains a death domain (DD), which is responsible for recruiting signaling molecules with DDs, including TNF receptor-associated factor 6 and TAK1 binding protein 1 and 2 . This recruitment induces the activation of $\mathrm{NF}-\kappa \mathrm{B}$-inducing kinase and the phosphorylation of the NF-kB inhibitor, I $\kappa B$ kinase (IKK1-IKK2-IKKK2). The activation of IKK promotes the ubiquitination and degradation of IкB- $\alpha$, resulting in the activation of NF- $\mathrm{B}, \mathrm{ERK}, \mathrm{p} 38$, JNK and other MAPKs, which then activate activator protein 1. Activation of this pathway induces the expression levels of IL-1, IL-6, IL-8, IL-12, TNF- $\alpha$ and other inflammatory cytokines (16).

Phosphoinositide 3-kinases (PI3Ks) are pivotal in inflammatory processes. In vivo and in vitro studies have demonstrated that leukocytes and macrophages lacking PI3K $\gamma$ exhibit defective chemotaxis towards various stimuli $(17,18)$. The inhibitory capacity of the selective inhibitor, AS041164, on peritoneal leukocyte chemotaxis towards RANTES is three times higher compared with that of LY294002 (19). LY294002 is a general PI3K inhibitor, whereas AS-041164 is a potent inhibitor of PI3K with selectivity for the class IB isoform PI3K $\gamma$. When administered orally to mice, AS-041164 demonstrates a pharmacokinetic profile that is similar to the general PI3K inhibitor LY294002 but is three times more potent at blocking neutrophil recruitment induced by RANTES. In addition, endothelial PI3K facilitates the adhesion of leukocytes to the inflammatory vessel wall (20). The expression of PI3K $\delta$ in endothelial cells promotes the adhesion of reactive leukocytes and the transmembrane migration of endothelial cells induced by TNF- $\alpha$ and leukotriene B4 (21). These observations demonstrate that PI3K $\delta$ and $\mathrm{PI} 3 \mathrm{~K} \gamma$ are required for the effective recruitment of white blood cells by endothelial cells in response to cytokine stimuli.

The present study established an in vitro model to examine the effects of N24 and p55PIK on the expression levels of TLR signaling pathway proteins in LPS-stimulated $\mathrm{HaCaT}$ cells. The $\mathrm{NF}-\kappa \mathrm{B}$ p65 protein translocation into the nucleus was altered upon LPS stimulation and N24 significantly suppressed this translocation. Furthermore, the ubiquitination and subsequent degradation of NF- $\kappa \mathrm{B}$ p 65 was observed in LPS-stimulated HaCaT cells. N24, but not p55PIK, significantly inhibited this ubiquitination and degradation, suggesting that N24 can effectively inhibit NF- $\kappa \mathrm{B}$ p65 translocation into the nucleus and prevent the release of pro-inflammatory cytokines. The expression levels of TLR2, TLR4 and MyD88 were demonstrated to increase with prolonged LPS stimulation in the HaCaT cells. The expression of $\mathrm{N} 24$ caused a reduction in the expression levels of TLR2, TLR4 and Myd88 in response to LPS stimulation. By contrast, p55PIK promoted the expression levels of TLR2, TLR4 and MyD88, suggesting that activation of PI3K increased the immune response to LPS, while N24 functioned as a PI3K inhibitor. The effects of N24 and p55PIK on the MAPK pathway, another TLR-mediated inflammatory signaling pathway, were also investigated. N24 and p55PIK caused no significant change to the phosphorylation of ERK1/2, JNK or p38, suggesting that N24 and p55PIK affect TLRs/MyD88-mediated signaling in an MAPK-independent manner.

In conclusion, the present study demonstrated the effect of N24 and p55PIK on TLR signaling and the suitability of PI3K as a potential novel target for immunosuppressant therapy and N24 as a novel candidate immunosuppressant. The underlying mechanism of $\mathrm{N} 24$ was also examined. These findings provide a theoretical basis for the identifcation of immunosuppressive drugs and therapeutic strategies against AID.

\section{Acknowledgements}

This study was supported by a grant from the National Science Foundation of China (no. 81072431).

\section{References}

1. Cho JH and Gregersen PK: Genomics and the multifactorial nature of human autoimmune disease. N Engl J Med 365: 1612-1623, 2011.

2. Bogdanos DP, Smyk DS, Rigopoulou EI, et al: Twin studies in autoimmune disease: genetics, gender and environment. J Autoimmun 38: J156-J169, 2012. 
3. Bruce B1 and Fries JF: The Stanford Health Assessment Questionnaire: a review of its history, issues, progress, and documentation. J Rheumatol 30: 167-178, 2003.

4. Bos JD and De Rie MA: The pathogenesis of psoriasis: Immunological facts and speculations. Immunol Today 20 40-46, 1999

5. Lowes M A, Kikuchi T, Fuentes-Duculan J, et al: Psoriasis vulgaris lesions contain discrete populations of Th1 and Th17 T cells. J Invest Dermatol 128: 1207-1211, 2008.

6. Cooper KD, Hammerberg C, Baadsgaard O, Elder JT, Chan LS, Sauder DN, Voorhees JJ and Fisher G: IL-1 activity is reduced in psoriatic skin. Decreased IL-1 alpha and increased nonfunctional IL-1 beta. J Immunol 144: 4593-4603, 1990.

7. Gomi T, Shiohara T, Munakata T, Imanishi K and Nagashima M: Interleukin 1 alpha, tumor necrosis factor alpha and interferon gamma in psoriasis. Arch Dermatol 127: 827-830, 1991.

8. Neuner P, Urbanski A, Trautinger F, Moller A, Kirnbauer R, Kapp A, Schopf E, Schwarz T and Luger TA: Increased IL-6 production by monocytes and keratinocytes in patients with psoriasis. J Invest Dermatol 97: 27-33, 1991.

9. Luger TA and Schwarz T: Evidence for an epidermal cytokine network. J Invest Dermatol 95: 100S-104S, 1990.

10. Gilhar A, Pillar T and Etzioni A: Possible role of cytokines in cellular proliferation of the skin transplanted onto nude mice. Arch Dermatol 131: 38-42, 1995.

11. Stein M, Bernd A, Ramirez-Bosca A, Kippenberger S and Holzmann H: Measurement of anti-inflammatory effects of glucocorticoids on human keratinocytes in vitro. Comparison of normal human keratinocytes with the keratinocyte cell line HaCaT. Arzneimittelforschung 47: 1266-1270, 1997.

12. Hu J, Xia X, Cheng A, Wang G, Luo X, Reed MF, Fojo T, Oetting A, Gong J and Yen PM: A peptide inhibitor derived from p55PIK phosphatidylinositol 3-kinase regulatory subunit: a novel cancer therapy. Mol Cancer Ther 7: 3719-3728, 2008.
13. Mutis T, De Bueger M, Bakker A and Ottenhoff TH: HLA class II+ human keratinocytes present Mycobacterium leprae antigens to CD4+ Th1-like cells. Scand J Immunol 37: 43-51, 1993.

14. Barker JN, Mitra RS, Griffiths CE, Dixit VM and Nickoloff BJ: Keratinocytes as initiators of inflammation. Lancet 337: 211-214, 1991.

15. Akira S: Toll-like receptors and innate immunity. Adv Immunol 78: 1-56, 2001.

16. Su SB, Silver PB, Grajewski RS, Agarwal RK, Tang J, Chan CC and Caspi RR: Essential role of the MyD88 pathway, but nonessential roles of TLRs 2, 4, and 9, in the adjuvant effect promoting Th1-mediated autoimmunity. J Immunol 175: 6303-6310, 2005.

17. Okkenhaug K, Bilancio A, Farjot G, Priddle H, Sancho S, Peskett E, Pearce W, Meek SE, Salpekar A and Waterfield MD, et al: Impaired $\mathrm{B}$ and $\mathrm{T}$ cell antigen receptor signaling in p110delta PI 3-kinase mutant mice. Science 297: 1031-1034, 2002.

18. Iwasaki A and Medzhitov R: Toll-like receptor control of the adaptive immune responses. Nat Immunol 5: 987-995, 2004.

19. Li Z, Jiang H, Xie W, Zhang Z, Smrcka AV and Wu D: Roles of PLC-beta2 and -beta3 and PI3Kgamma in chemoattractant-mediated signal transduction. Science 287: 1046-1049, 2000.

20. Puri KD, Doggett TA, Huang CY, Douangpanya J, Hayflick JS, Turner M, Penninger J and Diacovo TG: The role of endothelial PI3Kgamma activity in neutrophil trafficking. Blood 106: 150-157, 2005.

21. Puri KD, Doggett TA, Douangpanya J, Hou Y, Tino WT, Wilson T, Graf T, Clayton E, Turner M and Hayflick JS, et al: Mechanisms and implications of phosphoinositide 3-kinase delta in promoting neutrophil trafficking into inflamed tissue. Blood 103: 3448-3456, 2004. 